\title{
Occurrence, Identification, and Antimicrobial Resistance Profiles of Extended-Spectrum and AmpC b-Lactamase-Producing Enterobacteriaceae from Fresh Vegetables Retailed in Gauteng Province, South Africa
}

Loandi Richter, Erika M. Du Plessis, Stacey Duvenage, and Lise Korsten

\begin{abstract}
Extended-spectrum b-lactamase (ESBL) and AmpC b-lactamase-producing Enterobacteriaceae are no longer restricted to the health care system, but represent increased risks related to environmental integrity and food safety. Fresh produce has been increasingly reported to constitute a reservoir of multidrug-resistant (MDR) potential human pathogenic Enterobacteriaceae. This study aimed to detect, identify, and characterize the antimicrobial resistance of ESBL/AmpC-producing Enterobacteriaceae isolates from fresh vegetables at point of sale. Vegetable samples (spinach, tomatoes, lettuce, cucumber, and green beans; $n=545$ ) were purchased from retailers in Gauteng, the most densely populated province in South Africa. These included street vendors, trolley vendors, farmers' market stalls, and supermarket chain stores. Selective enrichment, plating onto chromogenic media, and matrix-assisted laser desorption ionization time-of-flight mass spectrometry (MALDITOF MS) confirmation of isolate identities showed that $17.4 \%$ (95/545) vegetable samples analyzed were contaminated with presumptive ESBL/AmpC-producing Enterobacteriaceae. Dominant species identified included Escherichia coli, Enterobacter cloacae, Enterobacter asburiae, and Klebsiella pneumoniae. Phenotypic antibiotic resistance analysis showed that $96.1 \%$ of 77 selected isolates were MDR, while resistance to aminoglycoside $(94.8 \%)$, chloramphenicol $(85.7 \%)$, and tetracycline $(53.2 \%)$ antibiotic classes was most prevalent. Positive phenotypic analysis for ESBL production was shown in 61 (79.2\%) of the 77 isolates, and AmpC production in $41.6 \%$ of the isolates. PCR and sequencing confirmed the presence of b-lactamase genes in $75.3 \%$ isolates from all vegetable types analyzed, mainly in E. coli, Enterobacter spp., and Serratia spp. isolates. CTXM group 9 (32.8\%) was the dominant ESBL type, while EBC (24.1\%) was the most prevalent plasmidic type AmpC b-lactamase. Our findings document for the first time the presence of MDR ESBL/AmpC-producing Enterobacteriaceae in raw vegetables sold at selected retailers in Gauteng Province, South Africa.
\end{abstract}

Keywords: antibiotic resistance, fresh produce, food safety

Introduction

corresponding isolates (Freitag et al., 2018). Since ESBL/AmpC b-lactamases are capable of inactivating broad-

Gxtended-spectrumb-lactamase (ESBL)- and AmpC-spectrum penicillins and cephalosporins, their presence in producing Enterobacteriaceae have increased in occur- Enterobacteriaceae is of clinical and epidemiological

rence globally in health care systems, agroecosystems, and fresh produce, due to the widespread use of broadspectrum antibiotics (Ye et al., 2017). Dissemination of these antimicrobial-resistant microorganisms has been identified as one of the six main antibiotic resistance (AR)-related health risks globally (WHO, 2015). If infection by ESBL/ AmpC-producing Enterobacteriaceae occurs, treatment options become limited as a result of expanded AR of the importance (Kolar et al., 2010). Clinically important ESBLproducing Enterobacteriaceae have been reported in different South African (SA) provinces (Eastern Cape [Vasaikar et al., 2017]; Western Cape [Peirano et al., 2011]; KwaZuluNatal [Mahomed and Coovadia, 2014]; and Gauteng Province [Ehlers et al., 2009]). In 53 clinical isolates from Gauteng, ESBL gene prevalence was reported in $87 \%$ (Ehlers et al., 2009).
ESBLs, classified as Ambler class A enzymes, include TEM-, SHV- and CTX-M-type enzymes (O” stholm, 2014; Ghafourian et al., 2015). More than 200 TEM and SHV variants have been documented, while 90 different enzymes within the CTX-M type have been described $\left(\mathrm{O}^{*}\right.$ stholm, 2014). Class A enzymes hydrolyze ampicillin and extendedspectrum cephalosporins (Ghafourian et al., 2015). AmpC blactamases, classified as class C enzymes, are 
resistant to additional b-lactams, that is, cephamycins, and are not influenced negatively by class A enzyme inhibitors (Jacoby, 2009; Njage and Buys, 2017). Plasmid-mediated AmpC (pAmpC)-producing strains are distinguished from chromosomal AmpC since they are often not inducible (Mezzatesta et al., 2012). Six families of pAmpC-blactamases, including

CIT, FOX, MOX, DHA, EBC, and ACC, have been described, with DHA, CMY (CIT family member), and FOX most commonly detected (Thomson, 2010). Co-occurrence of b-lactamase enzymes, especially AmpC b-lactamases and ESBLs, is common (Thomson, 2010).

Salmonella spp., pathogenic Escherichia coli, and Shigella spp. have been implicated in foodborne disease outbreaks, while Klebsiella pneumoniae, Serratia marcescens, Citrobacter freundii, and Enterobacter spp. are regarded as opportunistic human pathogenic bacteria (Baylis et al., 2011). The presence of ESBL/AmpC-producing Enterobacteriaceae on fresh produce has been studied worldwide (Kim et al., 2015; Nüesch-Inderbinen et al., 2015; Zurfluh et al., 2015).

Transfer of multidrug-resistant (MDR) Enterobacteriaceae onto fresh produce occurs through the use of contaminated irrigation water or during production via animal manure (van Hoek et al., 2015). Subsequent transfer to humans can happen through consumption of raw vegetables, potentially impacting consumer health negatively (Ye et al., 2017). Concomitantly AR genes can easily be transferred to commensal bacteria that typically colonize the human gut.

Fresh vegetables produced in SA are retailed nationally and to the South African Development Community (SADC) countries, Swaziland, the UK, Middle East, and Asian markets (DAFF, 2012a, b, 2016). Current knowledge regarding the occurrence of ESBL/AmpC-producing Enterobacteriaceae on fresh vegetables in SA is limited. The aim of this exploratory study was to detect, identify, and characterize the AR of ESBL- and AmpC-producing Enterobacteriaceae isolates from frequently consumed fresh vegetables from selected retailing sites in Gauteng, the most densely populated province in SA.

Materials and Methods

Sample collection

A total number of 545 vegetable samples was collected from 10 formal retailers, 10 street trading greengrocers, 10 mobile trolley vendors, and 13 vendors at two farmers' markets in Gauteng, SA, from September 2017 to May 2018 (Supplementary Fig. S1). In the informal markets, street traders typically display fresh produce on a table, underneath a shade covering, at the roadside, or they use mobile trolleys. The vegetable samples included, depending on availability, spinach (bunches, baby leaves, or minimally processed ready-to-eat [RTE] pillow packs; $n=200)$, tomatoes $(n=200)$, cucumbers $(n=45)$, lettuce (Iceberg lettuce heads or mixed salad leaf RTE pillow packs; $n=50)$, and green beans $(n=50$ samples). All samples were transported in cooler boxes and stored at $4 \mathrm{C}$ until further processing within $24 \mathrm{~h}$.

\section{Processing of fresh produce}

At least three leaves from one spinach bunch and the inner leaves of three lettuce heads were used to prepare $50 \mathrm{~g}$ composite samples of each of the leafy vegetable samples. Each spinach or lettuce sample was aseptically cut into a sterile polyethylene strainer stomacher bag containing $200 \mathrm{~mL}$ buffered peptone water (BPW) (3M, Johannesburg, $\mathrm{SA}$ ) in a 1:4 weight-to-volume ratio. A $150 \mathrm{~g}$ sample of tomatoes and cucumbers (composite of at least three tomatoes or cucumbers) and a $150 \mathrm{~g}$ sample of green beans were each placed into a sterile polyethylene stomacher bag containing $150 \mathrm{~mL}$ BPW in a 1:1 weight-to-volume ratio $(\mathrm{Xu}$ et al., 2015). Individual vegetable samples were blended for $5 \mathrm{~min}$ at $230 \mathrm{rpm}$ in a Stomacher 400 circulator paddle blender (Seward Ltd., London, United Kingdom).

Isolation and identification of presumptive extended-

spectrum and AmpC b-lactamase-producing

Enterobacteriaceae

Each of the BPW-sample mixtures was incubated for 3$4 \mathrm{~h}$ at $37 \mathrm{C}$ after which $1 \mathrm{~mL}$ of each sample was added to 9 $\mathrm{mL}$ Enterobacteriaceae enrichment broth (Oxoid, Johannesburg, SA) according to ISO 21528-1:2004 and incubated overnight at 30C (Blaak et al., 2014). ESBLproducing microorganisms were detected by streaking $10 \mathrm{lL}$ of each of the enriched samples onto ChromID ESBL agar plates (bioMe'rieux, Midrand, SA) and incubated overnight at 30C (Blaak et al., 2014). All presumptive positive ESBL/AmpC-producing Enterobacteriaceae colonies based on colony color, including weakly colored colonies, on the chromogenic media were isolated and purified.

Isolate identities were determined using matrix-assisted laser desorption ionization time-of-flight mass spectrometry (MALDI-TOF) (Bruker, Bremen, Germany) to species level as described by Standing et al. (2013). A single colony on nutrient agar was transferred to the MALDI-TOF polished steel target plate and further analyzed according to manufacturer's instructions (AOAC-OMA\#2017.09), following calibration with the bacterial test standard. NonEnterobacteriaceae isolates were not included in further analysis.

\section{Antimicrobial susceptibility testing}

A selection of 77 presumptive ESBL-producing Enterobacteriaceae isolates, representing all unique species per product type from each supplier, were selected for further analysis. The Kirby-Bauer disk diffusion technique 
was used to determine the resistance patterns of the isolates (Clinical Laboratory Standard Institute [CLSI], 2018). All isolates were screened for ESBL production by the doubledisk synergy test (DDST) using cefotaxime 301g, ceftazidime $301 \mathrm{~g}$, and cefpodoxime $101 \mathrm{~g}$, alone or in combination with clavulanic acid 101g (Mast Diagnostics, Randburg, SA) (EUCAST, 2013). Zone diameters were compared with the CLSI and EUCAST criteria to determine if isolates were 

resistant, intermediate, or susceptible. Isolates showing resistance to cefoxitin and cefotaxime or ceftazidime were regarded as a phenotypic indicator of AmpC production (EUCAST, 2013). Production of ESBLs was confirmed using the cefepime ESBL disc set (Cefepime 301g, cefepimeclavulanic acid 30-10lg) and AmpC production using the AmpC detection set (Mast Diagnostics) (EUCAST, 2013; CLSI, 2018). Additional antimicrobials tested for resistance or susceptibility of isolates included ampicillin 10lg, amoxicillin-clavulanic acid 20/10lg, amoxicillin $101 \mathrm{~g}$, trimethoprim-sulfamethoxazole $1.25 / 23.75 \mathrm{lg}$, imipenem $10 \mathrm{lg}$, neomycin $10 \mathrm{lg}$, tetracycline 30lg, gentamycin 101g, chloramphenicol 101g (Mast Diagnostics) (CLSI, 2018). Isolates resistant to three or more antimicrobial classes were regarded as MDR. $K$. pneumoniae ATCC 700603, E. coli NCTC 13315, Enterobacter cloacae NCTC 1406, and E. coli ATCC 25922 were included as positive and negative controls as described by the manufacturer (Mast Diagnostics).

\section{Characterization of b-lactamase genes}

The presence of ESBL determinants (bla $a_{\mathrm{TEM}}, b l a_{\mathrm{SHV}}$, bla $\left._{\mathrm{CTX}-\mathrm{M}}, b a_{\mathrm{OXA}}\right)$ and $\mathrm{pAmpC}$ resistance genes (bla $a_{\mathrm{ACC}}$, bla $_{\mathrm{FOX}}$, bla $_{\mathrm{MOX}}$, bla $_{\mathrm{DHA}}$, bla $_{\mathrm{CIT}}$, bla $\left._{\mathrm{EBC}}\right)$ in the selected isolates was analyzed with PCR and sequencing. Single colonies of each presumptive ESBL-producing Enterobacteriaceae isolate were cultured aerobically under shaking conditions at 200rpm in Tryptone soy broth (MERCK, Johannesburg, SA) for $24 \mathrm{~h}$ at $30 \mathrm{C}$. The cells were pelleted by centrifugation $(12,500 \mathrm{~g}$ for 10min), DNA was extracted using the QuickgDNA Mini-Prep kit (Zymo Research, Irvine, CA), and the DNA concentration was determined using the Qubit dsDNA

Broad Range Assay and a Qubit 2.0 fluorometer (Life Technologies, Johannesburg, SA). PCR was performed using the DreamTaq Green PCR Master Mix (ThermoFisher Scientific, Johannesburg, SA), specific primers, and thermocycling conditions for each of the genes as described in Supplementary Table S1.

PCR products were sequenced using BigDye Terminator v3.1 cycle sequencing on an ABI 3500XL sequencer in forward and reverse directions (InquabaBiotec, Johannesburg, SA). The sequences were edited with Chromas 2.6 and BioEdit sequence alignment editor software, and consensus sequences were subjected to BLAST nucleotide search analysis to identify the AR genes.

\section{Results}

Identification of presumptive extended-spectrum and

AmpC b-lactamase-producing

Enterobacteriaceae isolates

Using MALDI-TOF analysis, $122(28.2 \%)$ of the 432 presumptive extended-spectrum/AmpC b-lactamase- producing isolates obtained from the fresh vegetable samples were confirmed as Enterobacteriaceae belonging to 10 genera. The 310 non-Enterobacteriaceae isolates were predominantly identified as Pseudomonas spp. The Enterobacteriaceae isolates were identified as Enterobacter spp. (28.7\%), including E. cloacae, Enterobacter asburiae, Enterobacter cowanii, and Enterobacter ludwigii; Serratia (18.9\%), including predominantly Serratia fonticola; E. coli (18\%); Klebsiella spp. (14.8\%), including K. pneumoniae and Klebsiella oxytoca; Rahnella aquatilis (9\%); Proteus spp. (4.9\%), including Proteus penneri and Proteus mirabilis; Citrobacter spp. (2.5\%), including Citrobacter farmeri and C. freundii; Kluyvera ascorbata (1.64\%); Achromobacter xylosixidans (1.6\%); and Raoultella ornithinolytica (0.8\%). Presumptive ESBL/AmpCproducing Enterobacteriaceae were isolated from the vegetable types tested.

\section{Phenotypic AR profiling}

All the 77 selected presumptive ESBL-producing Enterobacteriaceae showed resistance to more than one antimicrobial agent, with $96.1 \%$ being MDR (resistant to $\$ 3$ antimicrobial classes) (Fig. 1). Resistance to the aminoglycosideandchloramphenicolclasseswasdominant,ob servedin

94.8\% and $85.7 \%$ oftheisolates, respectively.Allisolateswith cephalosporin resistance (CTX30C, CAZ30C, CPD10C, or CPM30C) were further screened using DDST, after which $61 / 77(79.2 \%)$ were tested positive for ESBL production (Fig. 1). All isolates that showed cefoxitin resistance $(n=46)$ were

additionallyscreenedwiththeAmpCdetectionset.Fromthese 46 isolates, 32/77 (41.6\%) were tested positive for AmpC production. This included 27 isolates showing resistance to cefoxitin, ceftazidime, and/or cefotaxime and additionally five isolates that showed cefoxitin resistance, but ceftazidime and/or cefotaxime susceptibility. All isolates displaying ESBL or AmpC phenotypes were further characterized for the identification of ESBL and/or AmpC resistance genes.

\section{Genotypic AR profiling}

Genes encoding b-lactamases were detected in 58/77 (75.3\%) isolates obtained from all vegetable types, mainly in E. coli $(n=20)$, Enterobacter spp. $(n=12)$, and Serratia spp. $(n=11)$ isolates. This included 37 (48\%) broadspectrum, 39 (51\%) ESBL, and 20 (25.9\%) AmpC genetic determinants (Fig. 1). The most frequently detected blactamase genes were bla $_{\mathrm{CTX}-\mathrm{M}}(n=28)$, followed by bla $a_{\mathrm{SHV}}$ $(n=22)$, bla $a_{\text {TEM }}(n=21)$, and bla ${ }_{\text {OXA }}(n=5)$. ESBLs encoded by

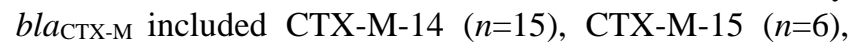
CTX-M-27 ( $n=4)$, and CTX-M-55 $(n=3)$; bla $a_{\text {TEM }}$ genes encoded TEM3 ( $n=3)$, while bla $a_{\text {SHV }}$ genes encoded SHV-18 
$(n=6)$, SHV28 $(n=1)$, and SHV-154 $(n=1)$. All the bla $a_{\text {OXA, }}$ $85.7 \%(n=18)$ of the $b l a_{\mathrm{TEM}}$, and $63.6 \%(n=14)$ of the bla $a_{\mathrm{SHV}}$

$\%$

FIG. 1. Summary of the species isolated from different fresh vegetables, indicating the phenotypic resistance profiles and the extended-spectrum b-lactamase/AmpC genetic determinants detected. The color code is given in the lower left corner of each section in grayscale: species identification (dark grey); isolate origin (black); phenotypic antimicrobial resistanceresistant (grey), intermediate resistant (light grey), or susceptible (white); genotypic determinants (black). AP10C, ampicillin; AUG30C, amoxicillin-clavulanic acid; A10C, amoxicillin; FOX30C, cefoxitin; CPM30C, cefepime; CPD10C, cefpodoxime; CPD10C/ CLAV1C, cefpodoxime-clavulanic acid; CAZ30C, ceftazidime; CAZ/CLAV10C, ceftazidimeclavulanic acid; CTX30C, cefotaxime; CTX/CLAV10C, cefotaxime-clavulanic acid; TS25C, trimethoprimsulfamethoxazole; IMI10C, imipenem; T30C, tetracycline; NE10C, neomycin; C10C, chloramphenicol. 


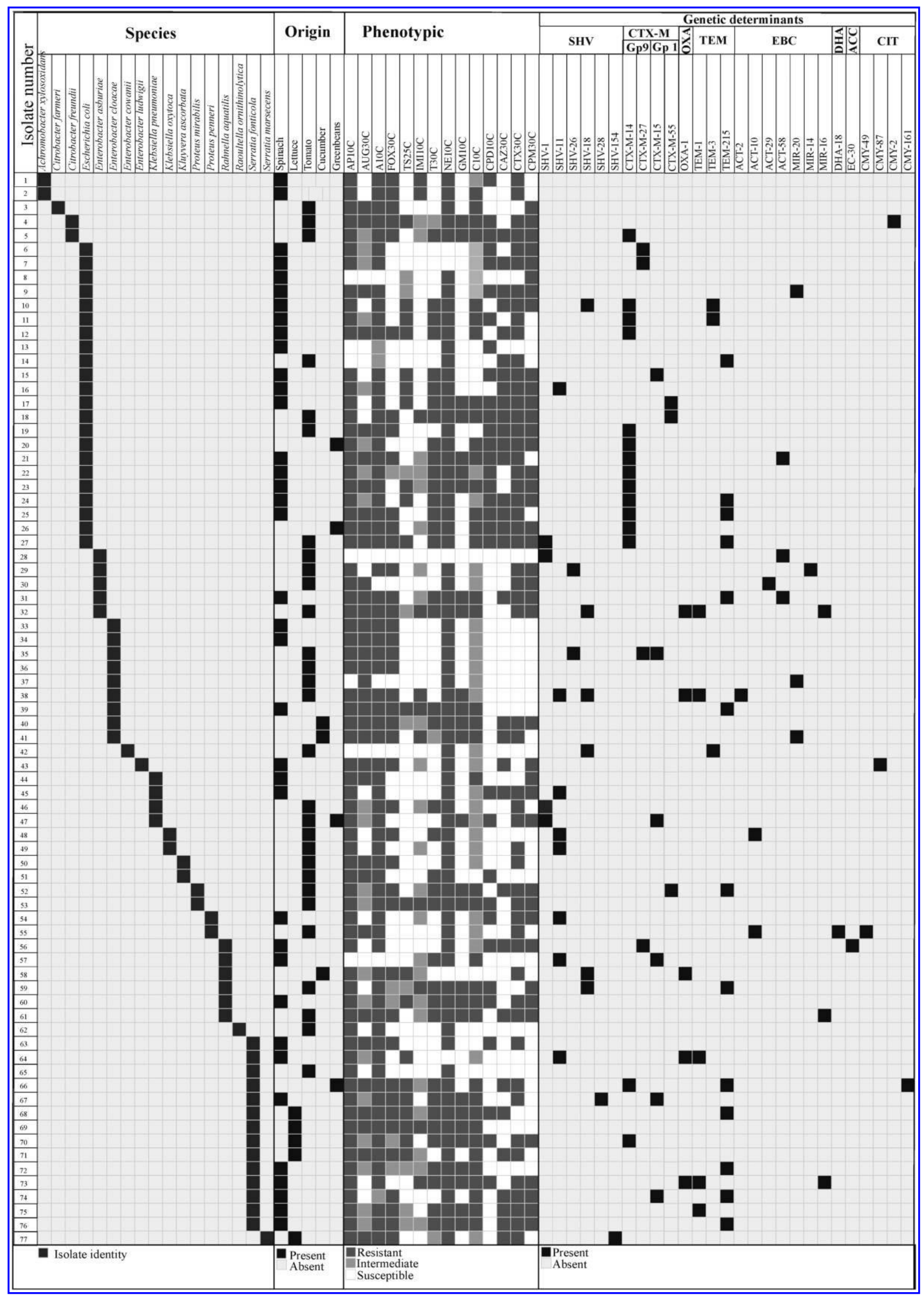


sequences encoded broad-spectrum b-lactamases OXA-1, TEM-1, TEM-215, SHV-1, SHV-11, or SHV-26, respectively. Three isolates harbored more than one ESBL; one

E. coli isolate carried the $b l a_{\mathrm{TEM}-3}, b l a_{\mathrm{SHV}-18}$, and $b l a_{\mathrm{CTX}-\mathrm{M} 14}$ genes, and two isolates (E. coli and E. cowanii) carried the blatem-3 gene in association with blactX-м-14 and blashv-18 genes, respectively. In 12 isolates (E. coli [n=3]; Enterobacter spp. [n=3]; Serratia spp. [n=3]; R. aquatilis [ $n=2]$; and P. mirabilis [ $n=1]$ ), ESBL genes in association with broad-spectrum b-lactamases were detected (Fig. 1).

AmpC resistance genes were detected in 18/58 (31\%) isolates harboring b-lactamase genetic determinants (Fig. 1). In 17 isolates, only one $\mathrm{pAmpC}$ genetic determinant was detected; blamir-20 $(n=4)$, blaмIR-16 $(n=3)$, blaACT-58 $(n=2)$, and one isolate each carried $b l a_{\mathrm{CMY}-2}, b l a_{\mathrm{MIR}-14}, b l a_{\mathrm{ACT}-29}$, blaACT-10, blaАCT-2, blaЕC, bla respectively. Among these 17 isolates, five isolates (Enterobacter spp. [n=2], E. coli [n=1], R. aquatilis [ $n=1]$, and $S$. fonticola $[n=1]$ ) also harbored ESBL genetic determinants. One P. penneri isolate carried three AmpC genes

$\left(b l a_{\mathrm{ACT} 10}, b l a_{\mathrm{DHA}-18}\right.$, and $\left.b l a_{\mathrm{CMY}-49}\right)$. The EBC family of the AmpC genetic determinants was the most dominant type.

\section{Discussion}

MDR ESBL/AmpC-producing Enterobacteriaceae were detected for the first time in raw vegetables retailed at selected sites in Gauteng Province, SA. Antibiotic-resistant opportunistic pathogens on fresh produce are a serious health concern that contributes toward the burden of AR in different environments, leading to increased risk of infection if colonization in humans occurs (Al-Kharousi et al., 2016). Enterobacteriaceae regarded as emerging bacterial threats include E. coli, K. pneumoniae, and Enterobacter spp. showing resistance to blactams and aminoglycosides (Fair and Tor, 2014).

Presumptive ESBL producers, predominantly E. coli, $K$. pneumoniae, E. cloacae, and E. asburiae, were detected in $17.4 \%$ of our vegetable samples analyzed. This is lower than the $25.4 \%$ reported by Zurfluh et al. (2015) for imported vegetables into Switzerland from the Dominican Republic, India, Thailand, and Vietnam, but higher than the 6\% reported by Reuland et al. (2014) on retail vegetables in the Netherlands. Similar to Blaak et al. (2014), environmental ESBL-producing Enterobacteriaceae isolated from vegetables included $S$. fonticola and $R$. aquatilis.

Phenotypic confirmation of ESBL/AmpC production showedthat61(79.9\%)ofthe77analyzedEnterobacteriaceae isolates displayed an ESBL-producing phenotype and 41.6\% an AmpC-producing phenotype, which is higher than results reported by van Hoek et al. (2015). Combined ESBLand producingphenotypeswerealsoobservedin $35 \%$ ofthe isolates. MDR phenotypes (resistance to $\$ 3$ antimicrobial classes) were observed in $96.1 \%$ of our analyzed isolates. The most prevalent non-b-lactam resistance profiles showed resistance against aminoglycoside (94.8\%), chloramphenicol (85.7\%), and tetracycline (53.2\%). This is higher than reports fromsimilarstudiesthatshowedresistancetoaminoglycosides (46.7-66.7\%), chloramphenicol (33.3\%) (Zurfluh et al., 2015; Ben Said et al., 2016), and tetracycline (46.7\%) (Ben Said et al., 2016) in ESBL-producing Enterobacteriaceae.

Genes expressing broad-spectrum b-lactamases, ESBLs, and/or AmpC b-lactamases were detected in $69.9 \%$ of our MDR isolates. Co-expression of ESBL and AmpC genes in environmental (van Hoek et al., 2015; Ye et al., 2017) and clinical (Tau et al., 2012; Kharat et al., 2017) Enterobacteriaceae isolates has also been reported. Globally the bla $a_{\text {СтX-M-type }}$ ESBL genes are predominant in Enterobacteriaceae, which was similar in our study, the majority being detected in E. coli isolates. bla $a_{\mathrm{CTX}-\mathrm{M}-14}$ was the main genetic determinant detected from mostly $E$. coli and $C$. freundii isolates, which corresponds to results obtained from vegetable samples in Tunisia (Ben Said $e t$ $a l ., 2016)$. Isolates harboring bla $a_{\mathrm{CTX}-\mathrm{M}-15}$ included E. coli, $E$. cloacae, K. pneumoniae, R. aqualtilis, and S. fonticola and were second most prevalent in our study. bla $a_{\text {СтХ-M-15 was }}$ the most prevalent gene detected in $E$. coli and $K$. pneumoniae isolates from fresh vegetables imported into Switzerland from India and the Dominican Republic (Zurfluh et al., 2015). This is in agreement with reports that bla $a_{\text {СТХ-М-14 }}$ and $b l a_{\text {СTX-M-15 }}$ are predominant and have been associated with clinically relevant Enterobacteriaceae infections (Ehlers et al., 2009; Zurfluh et al., 2015).

In contrast to Njage and Buys (2014), who predominantly detected bla $_{\text {CTX-M Group 8/25-positive } E \text {. coli isolates from lettuce }}$ in the North West Province (SA), no bla $a_{\text {CTX-M Group } 8 / 25 \text { genes }}$ were detected in any of our $E$. coli isolates from the vegetable samples analyzed. The bla $a_{\text {CTX-M-15 }}$ (CTX-M group 1) and bla $_{\text {CTX-M-14 }}$ (CTX-M group 9) genes detected in our environmental isolates, reported to be closely related to chromosomally encoded bla $a_{\mathrm{FONA}}$ and bla $a_{\mathrm{RAHN}}$ genes of $S$. fonticola and $R$. aquatilis, had no significant similarity in the GenBank database using NCBI BLAST based on total BLAST alignment scores. This contrasts results reported by Raphael et al. (2011) where sequences similar to bla $a_{\mathrm{RAHN}-2}$ and $b l a_{\mathrm{FONA}-5}$ were detected using $b l a_{\text {CTX-м }}$ primers.

In our study, five isolates, including E. coli, Enterobacter spp., $R$. aquatilis, S. fonticola, simultaneously harbored ESBL and $\mathrm{AmpC}$ genes. Environmental isolates are known to carry chromosomally encoded AmpC b-lactamases. However, Enterobacteriaceae harboring both chromosomal and $\mathrm{pAmpC}$ b-lactamases are increasingly reported to hydrolyze broadspectrum cephalosporins more efficiently, resulting in adverse treatment options in clinical settings (Jacoby, 2009; Reuland et al., 2014). 
The 18 isolates in which $\mathrm{pAmpC}$ resistance genes were detected predominantly included the EBC-type pAmpC blactamases (identified as $b l a_{\mathrm{ACT}} / b l a_{\mathrm{MIR}}$ ). This contrasts with two previous studies where $b l a_{\mathrm{CIT}}, b l a_{\mathrm{DHA}}$, or $b l a_{\mathrm{ACC}} \mathrm{pAmpC}$ b-lactamases were mostly detected in Enterobacteriaceae isolated from fresh produce and water samples (Njage and Buys, 2014; Ye et al., 2017). bla $a_{\mathrm{ACT} / \mathrm{MIR}}$ genes have been reported tobe thedominantAmpCgenetic determinantsinEnterobacter spp., causing intra-abdominal infections (Khari et al., 2016), and were detected in seven of the Enterobacter spp. isolates in our study. The fact that fresh produce can serve as a reservoir of MDR ESBL/AmpCproducing Enterobacteriaceae, including their genetic determinants, constitutes a potential health risk to the consumer as resistance to antimicrobials frequently used to treat human infections was shown.

\section{Conclusion}

The results obtained from screening at these selected sites

\section{6}

indicate that further investigation of different fresh produce types in Gauteng and other provinces in SA is necessary. Future studies should focus on the surveillance of production systems from farm to retail to identify potential sources of contamination that contribute to the presence and dissemination of antimicrobial-resistant microorganisms and their genetic determinants. Since AR is a worldwide problem, a global solution is required that integrates the contributions from government departments as well as from the scientific community.

\section{Acknowledgments}

The authors would like to acknowledge the financial assistance of the Water Research Commission in SA and the Department of Science and Technology-National Research Foundation (NRF), Centre of Excellence in Food Security. MALDI-TOF analysis was based on the research supported in part by the NRF (grant specific reference number (UID) 74426). Conclusions arrived at are those of the authors and are not necessarily to be attributed to the NRF. The authors would like to acknowledge Dr. Germa'n Villamizar-Rodri'guez for guidance with genotypic characterization, and Ms. Zama Zulu for assistance with MALDI-TOF identification.

\section{Disclosure Statement}

No competing financial interests exist.

Supplementary Material

Supplementary Figure S1

Supplementary Table S1

\section{References}

Al-Kharousi ZS, Guizani N, Al-Sadi AM, Al-Bulushi IM, Shaharoon B. Hiding in fresh vegetables: Opportunistic pathogens may cross geographical barriers. Int J Microbiol 2016;1-14. DOI: 1-.1155/2016/4292417.

Baylis C, Uyttendaele M, Joosten H, Davies A, Heinz HJ. The Enterobacteriaceae and their significance to the food industry. ILSI Europe Report Series 2011;1-48.
Ben Said L, Klibi N, Dziri R, Borgo F, Boudabous A, Ben Slama K, Torres C. Prevalence, antimicrobial resistance and genetic lineages of Enterococcus spp. from vegetable food, soil and irrigation water in farm environments in Tunisia. J Sci Food Agric 2016;96:1627-1633.

Blaak H, van Hoek AHAM, Veeman C, Docters van Leeuwen AE, Lynch G, van Overbeek WM, de Roda Husman AM.

Extended spectrum b-lactamase- and constitutively AmpCproducing Enterobacteriaceae on fresh produce and in the agricultural environment. Int $\mathrm{J}$ Food Microbiol 2014;168- 169:8-16.

Dallenne C, Da Costa A, Decre' D, Favier C, Arlet G. Development of a set of multiplex PCR assays for the detection of genes encoding important beta-lactamases in Enterobacteriaceae. J Antimicrob Chemother 2010;65:490-495.

CLSI. Performance Standards for Antimicrobial Susceptibility Testing. 28th ed. CLSI Supplement M100. Wayne, PA:

Clinical and Laboratory Standards Institute, 2018.

\section{RICHTER ET AL.}

Available at: www.clsi.org

Department of Agriculture, Forestry and Fisheries. A profile of the South African cucumber market value chain. South Africa: DAFF, 2012a:1-29.

Department of Agriculture, Forestry and Fisheries. A profile of the South African tomato market value chain. South Africa: DAFF, 2012b:1-34.

Department of Agriculture, Forestry and Fisheries. A profile of the South African lettuce market value chain. South Africa: DAFF, 2016:1-31.

Ehlers MM, Veldsman C, Makgotlho EP, Dove MG, Hoosen AA, Kock MM. Detection of bla $a_{\mathrm{SHV}}, b l a_{\mathrm{TEM}}$ and $b l a_{\mathrm{CTX}-\mathrm{M}}$ antibiotic resistance genes in randomly selected bacterial pathogens from the Steve Biko Academic Hospital. FEMS Immunol Med Microbiol 2009;56:191-196.

EUCAST. EUCAST guidelines for detection of resistance mechanisms and specific resistance of clinical and/or epidemiological importance 2013:1-43. DOI: 10.7150/ijbs. 13498.

Fair RJ, Tor Y. Perspectives in medicinal chemistry antibiotics and bacterial resistance in the 21st Century. Perspect Med Chem 2014;6:25-64.

Freitag C, Michael GB, Jun L, Kadlec K, Wang Y, Hassel M, Schwarz S. Occurrence and characterisation of ESBLencoding plasmids among Escherichia coli isolates from fresh vegetables. Vet Microbiol 2018;219:63-69.

Ghafourian S, Sadeghifard N, Soheili S, Sekawi Z. Extended spectrum beta-lactamases: definition, classification and epidemiology. Curr Issues Mol Biol 2015;17:11-22. Jacoby GA. AmpC beta-lactamases. Clin Microbiol Rev 2009; 22:161-182.

Kharat AA, Kharat KR, Chaudhari SG, Kadam DG, Kharat AS. Co-existence of multiple B-lactamase traits among clinical isolates of Escherichia coli from rural part of Maharashtra, India. Afr J Microbiol Res 2017;11:278-286.

Khari FIM, Karunakaran R, Rosli R, Tay ST. Genotypic and phenotypic detection of AmpC b-lactamases in Enterobacter spp. Isolated from a teaching hospital in Malaysia. PLoS One 2016;11:1-12.

Kim HS, Chon JW, Kim YJ, Kim DH, Kim MS, Seo KH. Prevalence and characterization of extended-spectrumbetalactamase-producing Escherichia coli and Klebsiella 
pneumoniae in ready-to-eat vegetables. Int J Food Microbiol 2015; 207:83-86.

Kolar M, Bardon J, Chroma M, Hricova K, Stosova T, Sauer P, Koukalova D. ESBL and AmpC beta-lactamase-producing Enterobacteriaceae in poultry in the Czech Republic. Vet Med 2010;55:119-124.

Mahomed S, Coovadia YM. Faecal carriage of Extended Spectrum Beta-lactamase producing Escherichia coli and Klebsiella pneumoniae in children from the community of Kwadedangendlale, KwaZulu-Natal, South Africa. Int J Infect Cont 2014;11:1-8.

Mezzatesta ML, Gona F, Stefani S. Enterobacter cloacae complex: Clinical impact and emerging antibiotic resistance. Future Microbiol 2012;7:887-902.

Njage PMK, Buys EM. Pathogenic and commensal Escherichia coli from irrigation water show potential in transmission of extended spectrum and AmpC b-lactamases determinants to isolates from lettuce. Microb Biotechnol 2014;8:462-473.

Njage PMK, Buys EM. Quantitative assessment of human exposure to extended spectrum and AmpC b-lactamases bearing $E$. coli in lettuce attributable to irrigation water and subsequent horizontal gene transfer. Int $\mathbf{J}$ Food Microbiol 2017; 240:141-151.

Nu“esch-Inderbinen M, Zurfluh K, Peterhans S, Hächler H, Stephan R. Assessment of the prevalence of extendedspectrum b-lactamase-producing Enterobacteriaceae in ready-to-eat salads, fresh-cut fruit, and sprouts from the Swiss market. J Food Prot 2015;78:1178-1181.

O" stholm A ${ }^{\circ}$ B. Extended-Spectrum b-Lactamase-Producing Enterobacteriaceae: Antibiotic Consumption, Detection and Resistance Epidemiology. Linko"ping, Sweden: Linkoping University, 2014.

Peirano G, van Greune CHJ, Pitout JDD. Characteristics of infections caused by extended-spectrum blactamaseproducing Escherichia coli from community hospitals in South Africa. Diagn Microbiol Infect Dis 2011;69:449-453.

Raphael E, Wong LK, Riley LW. Extended-spectrum betalactamase gene sequences in gram-negative saprophytes on retail organic and nonorganic spinach. Appl Environ Microbiol 2011;77:1601-1607.

Reuland EA, al Naiemi N, Raadsen SA, Savelkoul PHM, Kluytmans JAJW, Vandenbroucke-Grauls CMJE. Prevalence of ESBL-producing Enterobacteriaceae in raw vegetables. Eur J Clin Microbiol Infect Dis 2014;33:18431846.

Standing TA, du Plessis EM, Duvenage S, Korsten L. Internalisation potential of Escherichia coli O157:H7, Listeria monocytogenes, Salmonella enterica subsp. enterica serovar Typhimurium and Staphylococcus aureus in lettuce seedlings and mature plants. J Water Health 2013;11:210-223.

Tau NP, Smith AM, Sooka A, Keddy KH. Molecular characterization of extended-spectrum b-lactamase producing Shigella isolates from humans in South Africa, 2003-2009. J Med Microbiol 2012;61:162-164.

Thomson KS. Extended-spectrum-beta-lactamase, AmpC, and carbapenemase issues. J Clin Microbiol 2010;48:1019-1025. van Hoek AHAM, Veenman C, van Overbeek WM, Lynch G, de Roda Husman AM, Blaak H. Prevalence and character- ization of ESBL- and AmpC-producing Enterobacteriaceae on retail vegetables. Int J Food Microbiol 2015;204:1-8.

Vasaikar S, Obi L, Morobe I, Bisi-Johnson M Molecular characteristics and antibiotic resistance profiles of Klebsiella isolates in Mthatha, Eastern Cape province, South Africa. Int J Microbiol 2017:1-7. DOI: 10.1155/2017/8486742.

WHO. Global Antimicrobial Resistance Surveillance System. Geneva: World Health Organisation, 2015.

$\mathrm{Xu}$ A, Pahl DM, Buchanan RL, Micallef SA. Comparing the microbiological status of pre- and postharvest produce from small organic production. J Food Prot 2015;78:1072-1080.

Ye Q, Wu Q, Zhang S, Zhang J, Yang G, Wang H, Huang J,

Chen M, Xue L, Wang J. Antibiotic-resistant extended spectrum b-lactamase- and plasmid-mediated AmpCproducing Enterobacteriaceae isolated from retail food products and the Pearl river in Guangzhou, China. Front Microbiol 2017;8:1-12.

Zurfluh K, Nu“esch-Inderbinen M, Morach M, Berner AZ, Hachler H, Stephan R. Extended-spectrum-betalactamaseproducing Enterobacteriaceae isolated from vegetables imported from the Dominican Republic, India, Thailand, and Vietnam. Appl Environ Microbiol 2015;81:3115-3120. 